\title{
The role of the oblique effect in the block-design selection process
}

\author{
DANIEL B. BERCH and MARK M. LEACH \\ University of Cincinnati, Cincinnati, Ohio
}

\begin{abstract}
When attempting to reproduce units of a stimulus pattern in block-design construction tasks, subjects take longer to find a correctly oriented, two-color, diagonally divided surface than a correctly oriented solid-color surface. To examine the possible contribution of the diagonal orientation judgment itself to this outcome, a delayed matching-to-sample task was employed in which subjects attempted to find a match for either a diagonally divided or a perpendicularly divided half-red/half-white square. Slower response times for the former provided evidence that the greater difficulty in matching the two-color portions of the stimulus patterns in standard block-design tasks may be partially attributable to the oblique effect.
\end{abstract}

In block-design construction tasks, such as that found in the Wechsler intelligence scales, examinees are shown square, red-and-white stimulus patterns and are asked to reproduce them on the top surfaces of red-and-white colored blocks. Each block contains two each of the following types of surfaces: solid red, solid white, and twocolor diagonally divided half red/half white. Royer (1977) generated an information-processing model of performance based on these kinds of tasks, in which he assumed that the total solution time for correctly reproducing a given design is determined by the sum of the times required to execute five independent processing operations. Among these, the "block-selection" operation pertains to the time required for selecting a surface from the alternatives presented by the block faces so that it matches a given mentally recoded stimulus unit (a perceived portion of the stimulus pattern that has been reprocessed into units like those of the various block faces). Experimental manipulations of relevant task parameters have shown that when attempting to find such a match, subjects take longer to select a correctly oriented two-color surface than a correctly oriented solid-color surface (Royer, 1977; Schorr, Bower, \& Kiernan, 1982).

Since there are four possible two-color surfaces from which to choose (two bits of uncertainty), and only two solid-color surfaces (one bit), and since response time increases directly with the amount of uncertainty, slower latencies for selecting two-color surfaces may be entirely attributable to this factor (Royer, 1977). It has also been suggested that the orientation judgment required to correctly match a two-color stimulus unit may in itself be

This paper is based in part on a senior honors thesis by Mark M. Leach. Portions of this research were presented at the 1989 annual meeting of the Midwestern Psychological Association, Chicago, IL. Correspondence should be addressed to Daniel B. Berch, Department of Psychology, Dyer Hall (ML 376), University of Cincinnati, Cincinnati, OH 45221. partially responsible for the longer selection times (Schorr et al., 1982). That is, with two-color surfaces, one does not simply have more alternatives from which to choose, one must select the correct orientation of the block face.

From an information-theoretic standpoint, selecting an appropriately oriented two-color surface should not be of any greater difficulty than selecting one of four solid colors [as with the original Kohs (1923) blocks that had solid red, solid white, solid blue, and solid yellow surfaces, along with diagonally divided half-red/half-white and half-blue/half-yellow surfaces]. However, since the selection of a correctly oriented two-color surface requires, in part, retention of the orientation of an oblique dividing line, this in itself could contribute to the slower selection times. That is, a good deal of evidence exists indicating that other kinds of tasks involving the discrimination of and/or memory for oblique lines tends to present special difficulties (Appelle, 1972; Royer \& Holland, 1975).

According to Royer and Weitzel (1977), however, memory demands are minimal in the block-design task. This is certainly true in the sense that under standardized testing conditions, the stimulus pattern remains visible while the examinee attempts to reproduce it. Nevertheless, the examinee must retain information regarding the oblique orientation ( / or $\backslash$ ) of any given two-color unit of the design for at least a second or two while searching for the correctly matching block surface. Even this brief interval may provide enough time for the oblique effect to become operative. In order to assess this possibility, we attempted to simulate the block-selection phase of block-design problems by giving subjects a delayed matching-to-sample task, in which they were required to match either a standard diagonally divided two-color surface or a perpendicularly divided two-color surface. Slower response times for the former would constitute evidence that the oblique effect is in part responsible for the slower selection times associated with two-color blocks. 


\section{METHOD}

\section{Subjects}

The subjects were 20 undergraduates ( 10 men and 10 women) from introductory psychology classes who participated for course credit.

\section{Apparatus and Stimuli}

Stimuli were presented via a Gerbrands three-field tachistoscope. Sample stimuli, drawn to the left of center on standard $4 \times 6$-in. T-scope cards, consisted of two orientation types: a diagonally divided half$\mathrm{red} / \mathrm{half}$-white square and a perpendicularly divided half-red/half-white square, each measuring $21 \times 21 \mathrm{~mm}$ (see Figure 1). There were four possible sample stimuli for each orientation type, varying in terms of the positions of the red and white colors. For each orientation condition, there were four alternative squares from which to select a match. These squares were drawn on cards in the format of a $2 \times 2$ matrix (to the right of center), with the horizontally aligned squares separated by a distance of $28 \mathrm{~cm}$ and the vertically aligned squares separated by a distance of $25 \mathrm{~cm}$ (both measured from the inner edges). The squares were numbered 1-4 as shown in the right-hand portions of Figure 1. Different cards were made up in order to include all possible permutations.

\section{Procedure}

The subjects were administered a delayed matching-to-sample task in which they had to select, from an array of four alternatives each, a match for either a diagonally divided or a perpendicularly divided square. Four practice trials were administered first in order to familiarize the subjects with the stimuli and procedure. Each subject then received a total of 192 trials-96 diagonal and 96 perpendicular-with each orientation type occurring in four successive alternating blocks of 48 trials each. Each of the four sample stimuli within the two orientation types appeared as a correct match 24 times, 6 times at each location in the matrix. Both the sequence of sample stimuli and the location of the correct match were randomized within trial blocks. On a given trial, the subjects were first asked to fixate a black dot that was displayed for $500 \mathrm{msec}$. Next, a sample stimulus appeared for $1 \mathrm{sec}$, im-
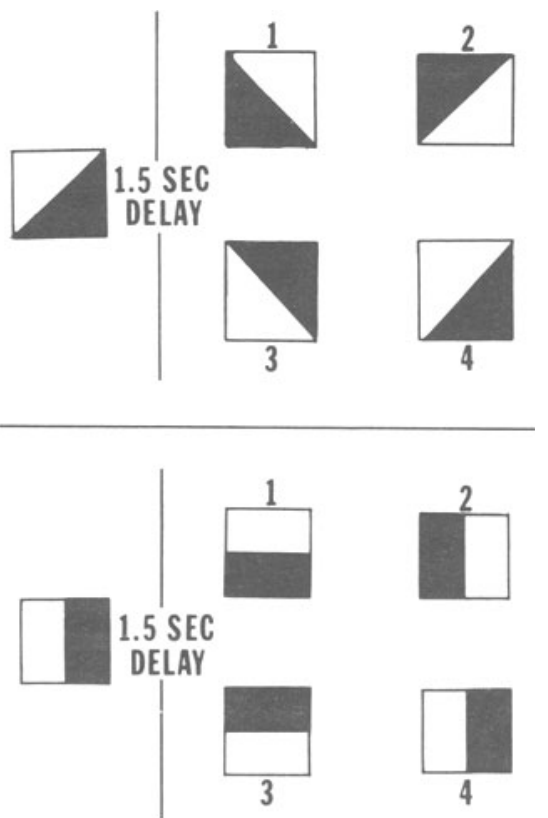

Figure 1. Sample trials from the diagonal (top) and perpendicular (bottom) orientation conditions. Actual stimuli were half red and half white. mediately followed by a 1.5 -sec delay period (with no stimulus present), and finally a card containing four numbered alte rnatives (the target and three nonmatching squares). The subjects responded by verbalizing one of the numbers. Response times (RTs), measured from the onset of the second card, were recorded in milliseconds by means of a gated digital clock timer triggered by a voice-activated relay.

\section{RESULTS AND DISCUSSION}

The mean overall error rate was $0.8 \%(1.0 \%$ and $0.5 \%$ for diagonal and perpendicular trials, respectively). Separate medians for diagonal and perpendicular trials were computed from each subject's RT data (for correct responses only). Means (and standard deviations) of these median RT values were 1,22l (145) and 1,141 (143) msec for diagonal and perpendicular trials, respectively. A related $t$ test yielded a significant difference between the two judgment types $[t(19)=: 4.07, p<.001]$, indicating that it took the subjects longer to select an appropriate match for diagonally divided surfaces than for perpendicularly divided surfaces. This outcome suggests that the greater difficulty in finding a match for a twocolor stimulus unit as compared with a solid-color unit in the standard block-design task cannot be attributed simply to differences in the information vilues associated with their respective block-face alternatives (i.e., two bits of uncertainty as compared to one). Apriarently, at least part of the difficulty stems from having to retain, however briefly, the orientation of the oblique line dividing the twocolor units of the stimulus patterns.

That the difference between the two conditions in the present study was "only" $80 \mathrm{msec}$ might indicate to some that the contribution of the oblique effect to slower selection times in the actual block-design task is negligible. To the contrary, even this small difference becomes magnified as the number of two-color units of the block-design pattern increases. In addition, although the four alternative block faces were simultaneously available for viewing in this experiment, they may not all be visible simultaneously in the actual task (i.e., on the top surfaces of the blocks, which are randomly tossed on the table before each problem). Thus, examinees may have to take additional time to turn over other blocks to find the appropriate two-color surface (not the most efficient strategy) or to rotate one two-color surface until the matching orientation is found. In either sase, that much more time is being taken during which the diagonal orientation of the coded stimulus unit may fail to be retained, or at least that the mental representation would be of such low quality that the examinee would have to take more time to update it by rechecking the appropriate portion of the stimulus pattern.

Furthermore, in the present experiment, the sample and alternatives were equal in area, whereas in the standard task, the smaller size of the stimulıs units corresponding to the larger block faces requires examinees to make a mental size transformation (Royer \& Weitzel, 1977). This processing operation would not only contribute more time 
to the overall solution time in general, but would require retention of the coded (or recoded) stimulus unit for that much longer, thereby increasing the likelihood that it would be forgotten.

In discussing the contribution of the block-selection operation to overall solution time, Royer (1977) noted that all of the response time hypothesized to be attributable to the pattern-recoding (stimulus-reprocessing) operation may actually be attributable to block selection. Although he cited evidence from his own experiment that is inconsistent with this notion, he nevertheless suggested that this possibility should be examined further. In addition, Royer was well aware that the oblique effect might operate in some manner in the block-design task. He pointed out that the diagonal orientation of the block faces tends to generate oblique patterns, and also that the axis of symmetry of the most difficult patterns is on one diagonal. He concluded, "An influence of this property of patterns should not be overlooked in the search for some understanding of the information processing of block design patterns" (p. 47). The present results provide direct evidence of just such an influence.

\section{REFERENCES}

APPELLE, S. (1972). Perception and discrimination as a function of stimulus orientation: The "oblique effect" in man and animals. Psychological Bulletin, 78, 266-278.

KoHs, S. C. (1923). Intelligence measurement: A psychological and statistical study based upon the Block-Design Test. New York: Macmillan.

ROYER, F. L. (1977). Information processing in the block design task. Intelligence, 1, 32-50.

Royer, F. L., \& Holland, T. R. (1975). Rotational transformation of visual figures as a clinical phenomenon. Psychological Bulletin, 82, 843-868.

RoYer, F. L., \& WeITzel, K. E. (1977). Effect of perceptual cohesiveness on pattern recoding in the block design task. Perception \& Psychophysics, 21, 39-46.

SChORR, D., Bower, G. H., \& Kiernan, R. (1982). Stimulus variables in the block design task. Journal of Consulting \& Clinical Psychology, 50, 479-487.

(Manuscript received February 16, 1990.) 